October 13, 2018

\title{
Breakdown of Hydrodynamics in a Simple One-Dimensional Fluid
}

\author{
Pablo I. Hurtado \\ Department of Physics, Boston University, Boston, Massachusetts 02215, USA, and \\ Institute Carlos I for Theoretical and Computational Physics, Universidad de Granada, Granada 18071, Spain
}

\begin{abstract}
We investigate the behavior of a one-dimensional diatomic fluid under a shock wave excitation. We find that the properties of the resulting shock wave are in striking contrast with those predicted by hydrodynamic and kinetic approaches, e.g., the hydrodynamic profiles relax algebraically toward their equilibrium values. Deviations from local thermodynamic equilibrium are persistent, decaying as a power law of the distance to the shock layer. Non-equipartition is observed infinitely far from the shock wave, and the velocity-distribution moments exhibit multiscaling. These results question the validity of simple hydrodynamic theories to understand collective behavior in $1 \mathrm{~d}$ fluids.
\end{abstract}

PACS numbers:

Most one-dimensional (1d) systems -both quantum and classical- display anomalous collective properties. Generally speaking, the dimensional constrain inherent in these systems affects strongly the propagation of fluctuations. These fluctuations, in turn, control the system cooperative behavior, so their abnormal propagation leads to the observed anomalies. This observation underlies most surprising results found in 1d [1, 2].

Particularly interesting is the nonequilibrium behavior of $1 \mathrm{~d}$ classical fluids [2, 3, 4, 5, 6, 7, 8, 9, 15, 16]. An essential feature of these systems is that the particle sequence remains unchanged during their time evolution. This important trait, also known as single-file constrain, is directly responsible for the anomalous propagation of fluctuations. For instance, in order for a particle in a $1 d$ fluid to move appreciably, a coherent, correlated motion of many particles is needed. This strongly suppresses diffusion: in $1 \mathrm{~d}$ (stochastic) fluids the mean square displacement grows now as $\left\langle\Delta x^{2}\right\rangle_{\mathrm{S} F}=2 F t^{1 / 2}$, much slower than usual Fickian diffusion $(\sim t)[3]$. This single-file diffusion, which turns out to be relevant in many fields ranging from membrane biophysics to carbon nanotubes, zeolites, DNA, polymers and nanofluids, etc., has been recently confirmed in experiments with colloids [3]. Common to all these systems is the presence of confining structures (e.g. narrow channels) inducing quasi-1d (single-file) motion.

In the same way anomalous diffusion reflects the strong spatial correlations in 1d fluids, energy transport in 1d reveals the presence of long-range velocity and current correlations. The simplest nonequilibrium situation where this is put forward is Fourier's law. When the 1d fluid is subject to a small temperature gradient $\nabla T$, an energy flux $J=-\kappa \nabla T$ appears from the hot to the cold reservoir. It has been found that the heat conductivity $\kappa$ has unusual large values for $1 \mathrm{~d}$ fluids. In particular, it is actually believed (though some controversy remains [4, 5]) that any classical 1d fluid with momentum-conserving interactions and non-zero total pressure should exhibit a divergent $\kappa$ in the thermodynamic limit, i.e. $\kappa \propto L^{\alpha}$ as the system size $L$ goes to $\infty$. Although there exist analytical and numerical evidences supporting this result in both 1d fluids and crystals, there is still no agreement on the theoretical framework and the exponent $\alpha>0$ : fluctuating hydrodynamics predicts $\alpha=1 / 3[\underline{6}$ ], while mode-coupling theories and Boltzmann equation result in $\alpha=2 / 5$ 7, 8], and numerical results are still inconclusive [4, 5] (see however 9] for new strong evidences supporting $\alpha=1 / 3$ ). In this way, the behavior of $1 \mathrm{~d}$ fluids far from equilibrium and the theoretical approach suitable for their description are still open problems.

In this Letter we study the response of a $1 \mathrm{~d}$ model fluid to a nonequilibrium excitation. In particular, we study the piston problem, a textbook example where we learn the notion of shock wave 10. Our results show that the anomalous nonequilibrium response of $1 \mathrm{~d}$ fluids cannot be described within a classical hydrodynamic approach. In particular, the two basic hypothesis underlying any hydrodynamic theory are violated in our model, namely: (i) perturbations away from local thermodynamic equilibrium decay algebraically, persisting for a long-time, and (ii) there are additional slowly-changing observables apart from the density, velocity, and energy fields.

Our model is the diatomic gas of hard-point particles [4, 5, 9]. In a line of length $L$ we introduce $N=L$ hardcore point particles interacting via elastic collisions. The particles have alternating masses, $m_{2 i-1}=m=1$ and $m_{2 i}=M, i \in\left[1, \frac{N}{2}\right]$. In addition, we include a moving piston of infinite mass, which starts moving from $x=0$ at $t=0$ with constant velocity $V=1$. All other particles are initially at rest (cold gas) 11], and start with random positions, provided that the alternating order holds. All simulations here reported correspond to systems with $N=2 \times 10^{4}$ particles (other sizes were also simulated to check our results against finite-size effects), and we average our results over $10^{4}-5 \times 10^{4}$ different realizations of the initial state.

The propagation of the piston into the cold fluid cre- 


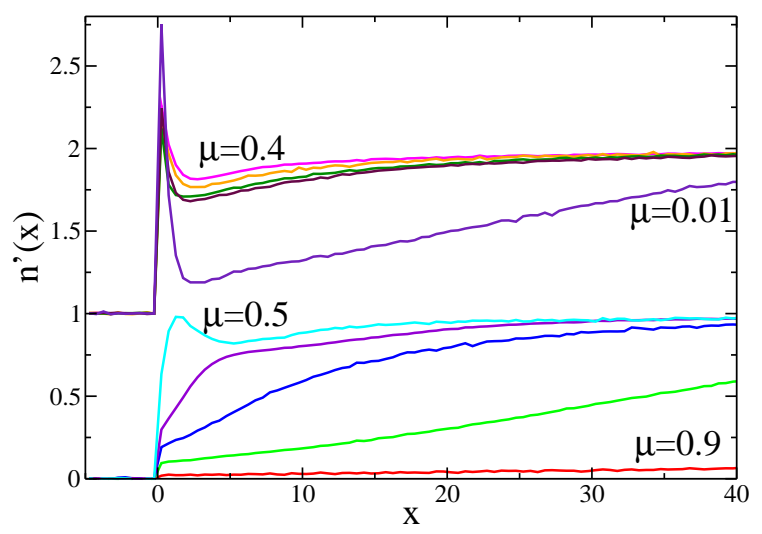

FIG. 1: (color online) Number density profiles for different mass ratios. From bottom to top, $\mu=0.9,0.8,0.7,0.618,0.5$. For $\mu<0.5$ profiles have been shifted 1 unit in the vertical axis. From top to bottom, $\mu=0.4,0.3,0.2,0.1,0.01$.

ates a shock wave traveling with velocity $W>V$. At any time $t$, the flow field consists of an aftershock region $V t<x<W t$ of excited gas attached to the piston, and a pre-shock region $x>W t$ of undisturbed gas. The position of the shock wave can be identified with the leading particle, trivially defined at any $t$ as the last particle disturbed from its initial, motionless state. For equal masses, $m=M$, the shock structure is particularly simple. In this case the system is equivalent to a $1 \mathrm{~d}$ gas of non-interacting particles: the leading particle moves with velocity $2 \mathrm{~V}$, and the aftershock fluid is composed by particles with velocity 0 and $2 \mathrm{~V}$, so the average is $V$. However, this limit is pathological, since the system is now non-ergodic, and lacks any relaxation mechanism in velocity space capable of driving the fluid toward (local) equilibrium. We may restore relaxation by letting $M>m$. In this case one expects to recover far behind the shock wave an equilibrium state with number density $n$, average flow velocity $V$ and temperature $T$. Conservation of particles, total momentum and total energy, or equivalently the constancy of the corresponding fluxes (Rankine-Hugoniot conditions), thus imply, 12]

$$
n=2 n_{0}, \quad W=2 V, \quad T=\frac{1}{2}(m+M) V^{2},
$$

where $n_{0}$ is the density of the undisturbed gas.

The randomness of the initial positions implies that the location and velocity of the shock wave (equivalently, the leader) are stochastic variables when consider within the ensemble of initial configurations. While the average shock position grows as $\left\langle x_{\ell}\right\rangle=W t$, the variance of the shock location is measured to grow diffusively, $\left\langle\Delta x_{\ell}^{2}\right\rangle=2 D t$, with $D$ an effective mobility (see 13. for similar shock fluctuations). To prevent these fluctuations from blurring the shock wave structure 14], we measure the flow profiles, characterizing the transition from the undisturbed gas toward the asymptotic equilibrium state (1), in a reference frame moving with the
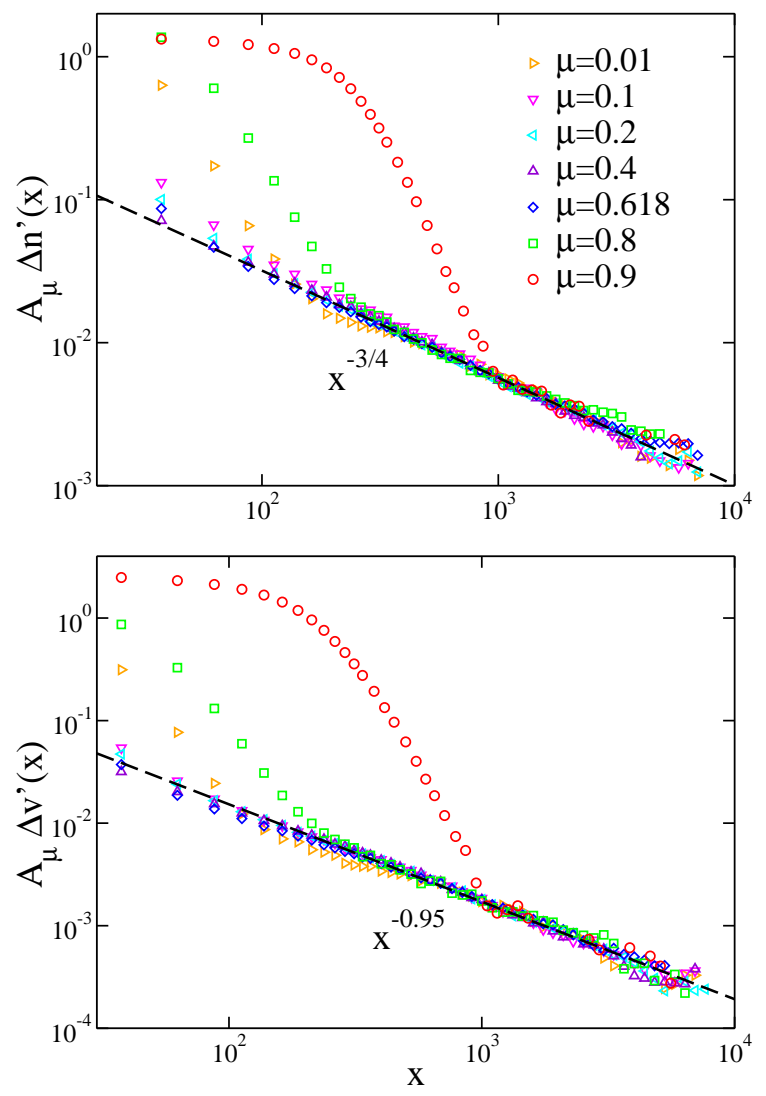

FIG. 2: (color online) Log-log scaling plot of $\Delta n^{\prime}(x)$ and $\Delta v^{\prime}(x)$. Many different $\mu \in[0.01,0.9]$ are included on each plot. The scaling factors $A_{\mu}$ have been selected in each case to fix the amplitude of the power-law tail to 1 .

leading particle. Moreover, profiles quickly converge to a steady shape after a short transient, thus allowing time averaging. Fig. 1 shows the normalized number density profiles measured in this way. Normalized observables are defined as $\phi^{\prime}(x)=\left(\phi(x)-\phi_{-}\right) /\left(\phi_{+}-\phi_{-}\right)$, where $\phi_{ \pm}=\phi(x \rightarrow \pm \infty)$.

Most interesting in Fig. 1] is the dependence of profiles on mass ratio, $\mu=m / M$. For $\mu$ close to 1 , the profile changes smoothly; in particular, the shock width (the length scale on which profiles change appreciably) is much larger than the mean free path. However, as $\mu$ decreases, the shock becomes steeper. This tendency lasts up to $\mu \approx 0.5$. At this point a density overshoot develops. Further decreasing $\mu$ results in a smoothening of the profile, with the exception of the initial overshoot, which sharpens as $\mu$ decreases. The shock width exhibits then a characteristic non-monotonous $\mu$-dependence, with a minimum around $\mu \approx 0.5$. Notice that $\mu \approx 0.5$ was previously reported to yield the fastest relaxation times for this model [5]. Similar behavior is observed in velocity and energy profiles (not shown). Interestingly enough, both density and velocity overshoots are not observed in hard-sphere fluids for $d>1$. 


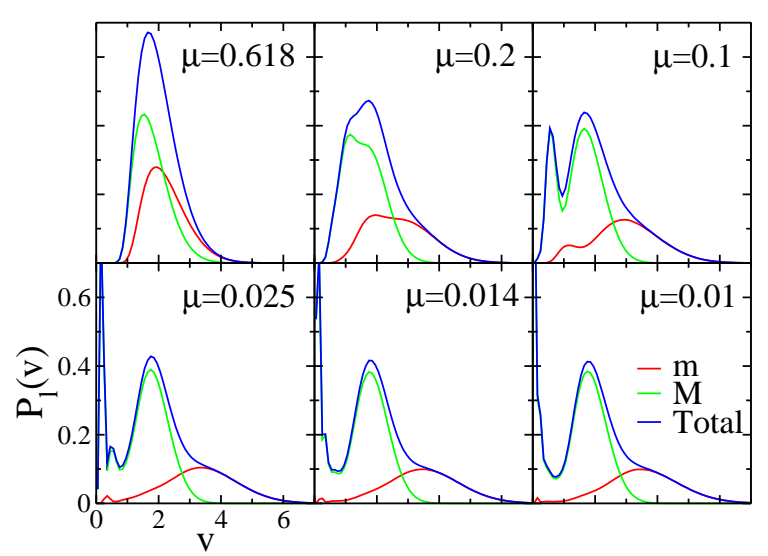

FIG. 3: (color online) $P_{\ell}(v)$ and $P_{\ell}^{(\mathrm{k})}(v), k=m, M$ for different mass ratios.

For large $x$, where $x$ is the distance from the shock layer, one expects the fluid to relax toward the equilibrium state (11). To characterize this relaxation, we now measure the excess flow fields as a function of $x$, defined as $\Delta \phi^{\prime}(x) \equiv 1-\phi^{\prime}(x)$, with $\phi^{\prime}=n^{\prime}, v^{\prime}, E^{\prime}$. This is shown in Fig. 2 It is remarkable that these observables relax toward the asymptotic state (1) as a power law, $\Delta \phi^{\prime}(x) \sim x^{-\beta_{\phi}}$. In particular, we find $\beta_{\mathrm{n}} \approx \frac{3}{4}$, and $\beta_{\mathrm{v}}=\beta_{\mathrm{E}} \approx 0.95 \neq \beta_{\mathrm{n}}$ (energy curves are not shown). These algebraic tails do not depend on $\mu$, as demonstrated by the scaling in Fig. 2] although the observed transient behavior is large for $\mu$ close to 0 and 1 , and minimal for $\mu \approx 0.5$. The power laws are a reflection of the large correlations present in 1d fluids, and are in striking contrast with the relaxation predicted by hydrodynamics and kinetic approaches, which is always exponential [10, 15]. In fact, this slow algebraic relaxation is related to the presence of long-wavelength hydrodynamic fluctuations [18], which control the fluid's transport properties. We discuss further this connection below.

We now focus on the shock wave propagation. Fig. 3 shows the shock stationary velocity distribution, $P_{\ell}(v) \equiv$ $P(x=0, v)$, for several $\mu$ 's. The average propagation velocity is $W=2$ in all cases, as expected. However, a multi-peaked structure in $P_{\ell}(v)$ emerges for $\mu \leq 0.5$, which coincides with the appearance of profile overshoots, see Fig. 11 This exemplifies how far the system is from local thermodynamic equilibrium near the shock layer. We may split $P_{\ell}(v)=P_{\ell}^{(\mathrm{m})}(v)+P_{\ell}^{(\mathrm{M})}(v)$, where $P_{\ell}^{(\mathrm{k})}(v)$ is the probability density function (pdf) for finding a particle of mass $k=m, M$ and velocity $v$ leading the shock (or equivalently, the pdf $P^{(\mathrm{k})}(x=0, v)$ for having a particle of mass $k=m, M$ at $x=0$ with velocity $v$ in a reference frame moving with the shock wave). These distributions, plotted also in Fig. 3] show that the shock wave propagates faster (slower) whenever a light (heavy) particle is leading. Also remarkable is the incipient singularity in $P_{\ell}(v)$ for small $v$ as $\mu \rightarrow 0$. This, together

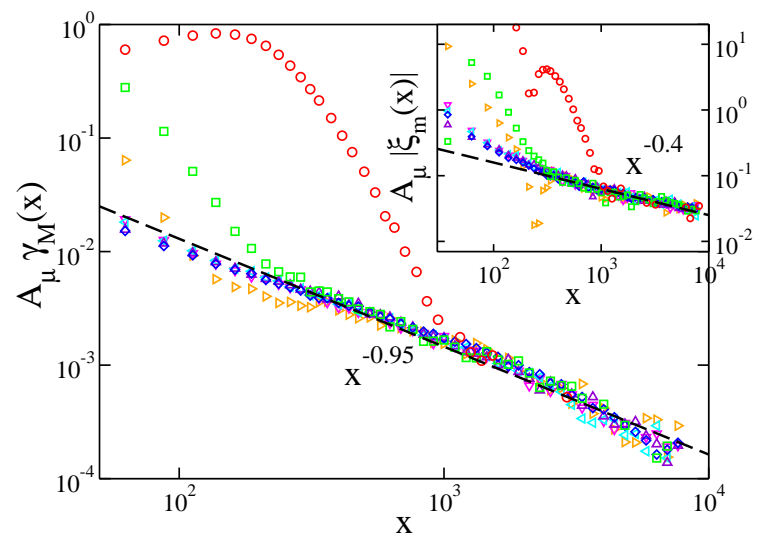

FIG. 4: (color online) Log-log scaling plot of $\gamma_{\mathrm{M}}(x)$ (main) and $\left|\xi_{\mathrm{m}}(x)\right|$ (inset) versus the distance to the shock. Definition of amplitudes $A_{\mu}$ and values of $\mu$ as in Fig. 2

with the singular overshoot in the profiles for small $\mu$ (Fig. 1), makes the limits $\mu \rightarrow 0$ and $\mu \rightarrow 1$ essentially different, contrary to recent claims [5].

Next, we turn our attention to the emergence of local thermodynamic equilibrium (LTE) behind the shock. For that, we measure the skewness $\gamma_{\mathrm{k}}(x)$ and kurtosis excess $\left|\xi_{\mathrm{k}}(x)\right|$ of the local velocity distribution for each species, $P^{(\mathrm{k})}(x, v), k=m, M$. They are defined as $\gamma_{\mathrm{k}}(x) \equiv$ $\left[\nu_{3}^{(\mathrm{k})}\left(\nu_{2}^{(\mathrm{k})}\right)^{-3 / 2}\right](x)$ and $\xi_{\mathrm{k}}(x) \equiv 3-\left[\nu_{4}^{(\mathrm{k})}\left(\nu_{2}^{(\mathrm{k})}\right)^{-2}\right](x)$, where $\nu_{i}^{(\mathrm{k})}(x)$ is the $i$-th central moment of $P^{(\mathrm{k})}(x, v)$. As the system reaches LTE behind the shock, both $\gamma_{\mathrm{k}}(x)$ and $\left|\xi_{\mathrm{k}}(x)\right|$ must go to zero. Fig. 4 shows the actual relaxation. Surprisingly, we find that both quantities decay also as slow power laws, $\phi_{\mathrm{k}}(x) \sim x^{-\beta_{\phi}}$, where now $\beta_{\gamma} \approx 0.95$ and $\beta_{\xi} \approx 0.4$ (exponents are independent of the species $k=m, M$ ). This proves that perturbations away from LTE are persistent in 1d, and that higherorder fields other than the hydrodynamic ones (namely, $\gamma_{\mathrm{k}}(x)$ and $\left.\left|\xi_{\mathrm{k}}(x)\right|\right)$ change slowly and therefore are relevant. Also interesting is the fact that $\beta_{\gamma}>\beta_{\xi}$. This observation, combined with the other exponents $\beta_{\mathrm{n}, v, E}$, implies that the distributions $P^{(\mathrm{k})}(x, v)$ exhibit multiscaling. This emerging picture is intriguing and exemplifies the anomalous behavior of $1 \mathrm{~d}$ fluids. Another interesting observable is the total energy stored in light and heavy particles at a given time $t, \epsilon_{\mathrm{k}}(t), k=m, M$. Energy equipartition in the asymptotic equilibrium state implies that $\epsilon_{\mathrm{k}}(t) \rightarrow \frac{1}{4}(3 k+\bar{k}) V^{2} \equiv \epsilon_{\mathrm{k}}(\infty)$ as $t \rightarrow \infty$, where $\bar{k}$ is the mass other than $k$. The inset to Fig. 5 shows the excess energies $\Delta \epsilon_{\mathrm{k}}(t)=\epsilon_{\mathrm{k}}(\infty)-\epsilon_{\mathrm{k}}(t), k=m, M$, for a particular $\mu$. They also decay as a power law, as expected from the algebraic tail of $\Delta E^{\prime}(x)$. However, what is remarkable is the different amplitudes: light particles are always closer to the asymptotic equipartition energy than heavy particles (i.e. light particles are more energetic than heavy ones [4, 15, 16, 17]). We may characterize this non-equipartition of energy with 


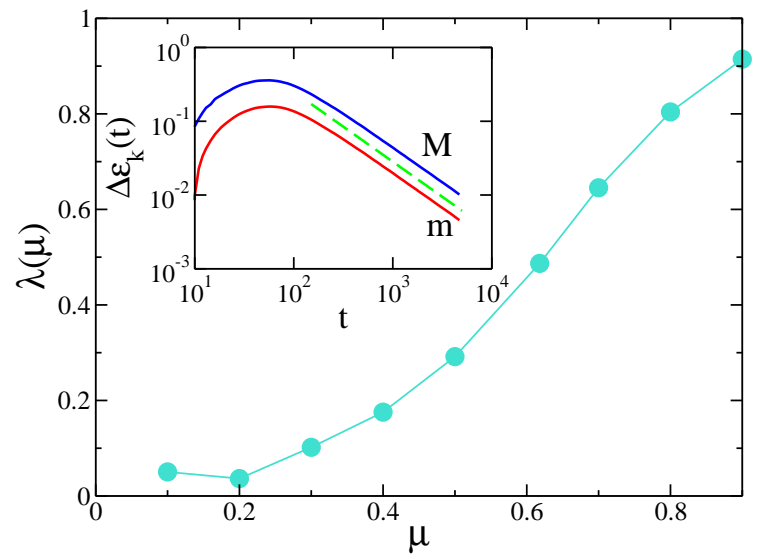

FIG. 5: (color online) Main: amplitude ratio $\lambda(\mu)$ (see main text for definition) as a function of $\mu$. Inset: Log-log plot of $\Delta \epsilon_{\mathrm{k}}(t), k=m, M$, vs. time for the particular case $\mu=0.8$. The dashed line has slope $0.95 \approx \beta_{\mathrm{E}}$.

the ratio $\lambda(\mu) \equiv \Delta \epsilon_{\mathrm{m}}(t) / \Delta \epsilon_{\mathrm{M}}(t)$, which converges to a $\mu$-dependent constant for long enough times. This is shown in Fig. 5 where it is evident that energy nonequipartition becomes more important as $\mu$ decreases.

A full theoretical explanation of the observed behavior seems challenging. Both hydrodynamic 10] and (Boltzmann) kinetic 15] approaches do not account for the measured shock structure and the slow, power-law relaxation of hydrodynamic observables. These algebraic tails result from long-wavelength hydrodynamic fluctuations, a main source of non-locality and long-range correlations in fluids. In fact, for $3 \mathrm{~d}$ fluids reflection and refraction of hydrodynamic fluctuations on a shock wave give rise to a slow, universal power-law relaxation of all hydrodynamic fields, $\Delta \phi(x) \sim x^{-3 / 2}, \phi=n, v, E$ [18]. Although somewhat similar in spirit, $1 \mathrm{~d}$ fluids exhibit a much more intricate behavior. The algebraic tails for the hydrodynamic fields are not universal, with exponents $\beta_{\mathrm{n}} \approx \frac{3}{4}$, and $\beta_{\mathrm{v}}=\beta_{\mathrm{E}} \approx 0.95 \neq \beta_{\mathrm{n}}$. In addition, we observe nonequipartition of energy and multiscaling behavior of oneparticle distribution functions. Most importantly, deviations away from local thermodynamic equilibrium are persistent, as characterized by the slow power-law decay of the skewness and kurtosis fields, with exponents $\beta_{\gamma} \approx 0.95$ and $\beta_{\xi} \approx 0.4$, respectively.

Any hydrodynamic description of collective behavior relies on two basic hypothesis [6]: (i) the system must reach local thermodynamic equilibrium rapidly (i.e. exponentially fast), and (ii) the only slowly-evolving observables are the density, velocity and energy fields. The slow algebraic relaxation of $\gamma_{\mathrm{k}}(x)$ and $\left|\xi_{\mathrm{k}}(x)\right|$ proves that both hypothesis are violated in our $1 \mathrm{~d}$ model fluid, which on the other hand contains the main ingredients characterizing a broad class of $1 \mathrm{~d}$ systems (i.e. dimensional constrain, and energy and momentum conservation). This suggests that the anomalous collective properties of $1 \mathrm{~d}$ fluids cannot be described within a simple hydrodynamic approach. This is particularly important in the study of transport phenomena in these systems, where hydrodynamic equations have been used to predict an anomalous thermal conductivity, for instance [6]. A sound theoretical approach to collective phenomena in $1 \mathrm{~d}$ fluids must therefore go beyond hydrodynamics. A good candidate is generalized kinetic theory. However, taking into account the strong inter-particle correlations present in 1d fluids in such a microscopic theory is a challenge that deserves further attention.

The author thanks P.L. Krapivsky for the original idea of this study, and T. Antal, P.L. Garrido, P.L. Krapivsky, and S. Redner for useful discussions. Financial support from the Spanish MEC is also acknowledged.

[1] V. Privman (ed.), Nonequilibrium Statistical Mechanics in One Dimension, Cambridge University Press, Cambridge (1997); T. Giamarchi, Quantum Physics in One Dimension, Oxford University Press, Oxford (2004).

[2] S. Lepri, R. Livi, A. Politi, Phys. Rep. 377, 1 (2003).

[3] T.E. Harris, J.Appl.Prob. 2, 323 (1965); D.G. Levitt, Phys.Rev. A 8, 3050 (1973); Q.-H. Wei et al, Science 287, 625 (2000); C. Lutz et al, Phys.Rev.Lett. 93, 026001 (2004); B. Cui et al, Phys.Rev.Lett. 89, 188302 (2002).

[4] P.L. Garrido et al, Phys. Rev. Lett. 86, 5486 (2001); P.L. Garrido, P.I. Hurtado, Phys. Rev. Lett. 88, 249402 (2002); ibid, Phys. Rev. Lett. 89, 079402 (2002).

[5] A. Dhar, Phys. Rev. Lett. 86, 3554 (2001); A.V.Savin et al, Phys. Rev. Lett. 88, 154301 (2002); P. Grassberger et al, Phys. Rev. Lett. 89, 180601 (2002).

[6] O. Narayan, S. Ramaswamy, Phys. Rev. Lett. 89, 200601 (2002).

[7] S. Lepri et al, Europhys. Lett. 43, 271 (1998); J.-S. Wang, B. Li, Phys. Rev. Lett. 92, 074302 (2004).

[8] A. Pereverzev, Phys. Rev. E 68, 056124 (2003).

[9] P. Cipriani et al, Phys. Rev. Lett. 94, 244301 (2005).

[10] G.B. Whitham, Linear and Nonlinear Waves, WileyInterscience 1999.

[11] This initial state ensures an inifinitely strong shock wave, which probes the fluid's whole nonequilibrium relaxation. A $T \neq 0$ initial state does not change our main results.

[12] la.B. Zeldovich, I.P. Raizer, Elements of Gasdynamics and the Classical Theory of Shock Waves, Academic Press, New York (1968).

[13] F.J. Alexander et al, Phys. Rev. E 47, 403 (1993).

[14] In this case, average profiles quickly converge to $\phi(x)=$ $1+\frac{1}{2} \operatorname{erfc}[x / \sqrt{2 D t}]$, the profile of a diffusing step.

[15] P.I. Hurtado, Phys. Rev. E 72, 041101 (2005); C. Cercignani, A. Frezzotti, P. Grosfils, Phys. Fluids 11, 2757 (1999).

[16] P.I. Hurtado, S. Redner, cond-mat/0507485

[17] A. Kato, D. Jou, Phys. Rev. E 64, 052201 (2001).

[18] A.F. Andreev, A.É. Meľerovich, Sov. Phys.-JETP 37, 829 (1973). 